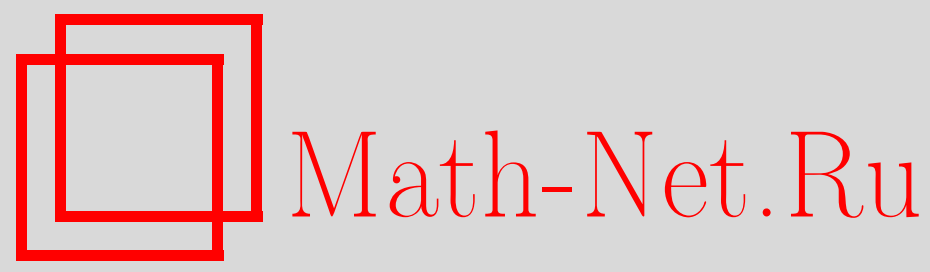

О. Ю. Шведов, О каноническом операторе Маслова в абстрактных пространствах, Матем. заметки, 1999, том 65, выпуск 3, 437-456

DOI: https://doi.org/10.4213/mzm1068

Использование Общероссийского математического портала Math-Net.Ru подразумевает, что вы прочитали и согласны с пользовательским соглашением http://www . mathnet.ru/rus/agreement

Параметры загрузки:

IP: 54.81 .137 .203

26 апреля 2023 г., 06:09:20

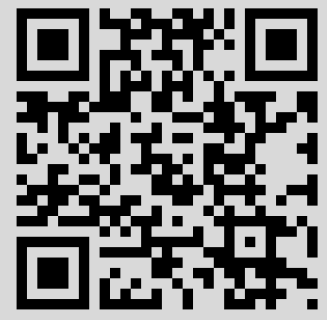




\section{О КАНОНИЧЕСКОМ ОПЕРАТОРЕ МАСЛОВА В АБСТРАКТНЫХ ПРОСТРАНСТВАХ}

\section{О. Ю. Шведов}

В последнее время был развит ряд новых методов построения асимптотических решений различных эволюционных уравнений. Эти асимптотики выражаются в каждый момент времени через элемент $y$ некоторого гладкого многообразия $\mathscr{Y}$ и элемент $f$ некоторого гильбертова пространства $\mathscr{F} y$. В работе исследуются некоторые общие свойства отображения, сопоставляющего паре $(y, f)$ асимптотическую формулу.

Библиографоия: 12 названий.

1. Введение. В квантовой механике часто используется квазиклассическое приближение. Одним из квазиклассических методов является теория комплексного ростка Маслова в точке [1], которая позволяет построить приближенные решения уравнений в частных производных с мальм параметром при операторе дифференцирования

$$
i \hbar \frac{\partial \psi}{\partial t}=H\left(t, x,-i \hbar \frac{\partial}{\partial x}\right) \psi, \quad t \in \mathbb{R}, \quad x \in \mathbb{R}^{n}, \quad \hbar \rightarrow 0,
$$

имеющие в каждый фиксированный момент времени $t$ вид волнового пакета с шириной порядка $\sqrt{\hbar}$ :

$$
\psi(x, t)=\frac{1}{\hbar^{n / 4}} \exp \left(\frac{i}{\hbar} S^{t}\right) \exp \left(\frac{i}{\hbar} P^{t}\left(x-Q^{t}\right)\right) f^{t}\left(\frac{x-Q^{t}}{\sqrt{\hbar}}\right),
$$

который отвечает движению классической частицы вдоль траектории $\left(P^{t}, Q^{t}\right) \in \mathbb{R}^{2 n}$.

В последнее время был развит [2]-[5] ряд методов построения асимптотических решений различных уравнений, не относящихся к типу (1), а имеющих более обший вид

$$
i \frac{\partial \psi^{\varepsilon}}{\partial t}=H^{\varepsilon} \psi^{\varepsilon} .
$$

Здесь $\psi^{\varepsilon}$-неизвестный элемент некоторого гильбертова пространства $\mathscr{H}^{\varepsilon}, H^{\varepsilon}$ - оператор в этом пространстве, $\varepsilon$ - устремляемьй к 0 малый параметр. В качестве примеров можно привести уравнение для системы $N$ частиц при $N \rightarrow \infty$ [2], уравнение для спиновых систем [3], уравнения квантовой теории поля [4], [5].

Построенные в [2]-[5] асимптотики решений уравнения (3) в каждьй момент времени $t$ выражаются через параметр $y$, принадлежащий некоторому гладкому многообразию $\mathscr{Y}$, и элемент $f$ гильбертова пространства $\mathscr{F}$. Асимптотическую формулу для решения уравнения (3), т.е. функцию $\psi_{\mathrm{as}}^{\varepsilon}(t)$, удовлетворяющую свойству

$$
\left\|\psi_{\text {as }}^{\varepsilon}(t)-\psi^{\varepsilon}(t)\right\| \rightarrow 0, \quad \varepsilon \rightarrow 0,
$$


можно представить через зависящий от $\varepsilon$ линейньй оператор $K_{y}^{\varepsilon}$, действующий из пространства $\mathscr{F}$ в пространство $\mathscr{H}^{\varepsilon}$ :

$$
\psi_{\text {as }}^{\varepsilon}(t)=K_{y(t)}^{\varepsilon} f(t) .
$$

Поскольку асимптотика (2) выражается через зависящие от $t$ параметры $(S, P, Q)$, $S \in \mathbb{R}, P, Q \in \mathbb{R}^{n}$, и элемент $f$ пространства $L^{2}\left(\mathbb{R}^{n}\right)$, формула $(2)$ является частньм случаем формулы (4) при $\mathscr{F}=L^{2}\left(\mathbb{R}^{n}\right), \mathscr{Y}=\mathbb{R}^{2 n+1}, \varepsilon=\hbar$,

$$
\left(K_{S, P, Q}^{\hbar} f\right)(x)=\frac{1}{\hbar^{n / 4}} \exp \left(\frac{i}{\hbar} S\right) \exp \left(\frac{i}{\hbar} P(x-Q)\right) f\left(\frac{x-Q}{\sqrt{\hbar}}\right) .
$$

Оператор $K_{y}^{\varepsilon}$, входящий в асимптотические формулы, построенные в [2]-[5], удовлетворяет следующим свойствам.

Свойство 1. При сдвиге параметра у на величину порядка в оператор $K_{y}^{\varepsilon}$ домножсается на регулярный при $\varepsilon \rightarrow 0$ числовой множитель $\chi_{y}(\delta y)$ :

$$
K_{y+\varepsilon \delta y}^{\varepsilon} f=\chi_{y}(\delta y) K_{y}^{\varepsilon} f+O(\sqrt{\varepsilon}) .
$$

Свойство 2. При сдвиге параметра у на величину порядка $\sqrt{\varepsilon}$ оператор $K_{y}^{\varepsilon}$ преобразуется следующим образом:

$$
K_{y+\sqrt{\varepsilon} \delta y}^{\varepsilon} f=\chi_{y}(\delta y) K_{y}^{\varepsilon} V_{y}[\delta y] f+O(\sqrt{\varepsilon})
$$

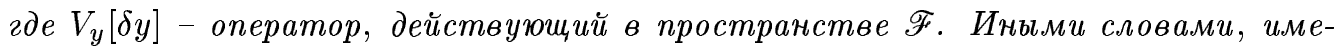
ет место инвариантность оператора $K_{y}^{\varepsilon}$ относительно сдвига параметра $у и$ преобразования аргумента этого оператора.

Свойства 1, 2 вьполнены для оператора (5), поскольку

$$
\begin{gathered}
K_{S+\hbar s, P+\hbar p, Q+\hbar q}^{\hbar} f=e^{i(s-P q)} K_{S, P, Q}^{\hbar} f+O(\sqrt{\hbar}), \\
K_{S+\sqrt{\hbar} s, P+\sqrt{\hbar} p, Q+\sqrt{\hbar} q}^{\hbar} f=\exp \left(\frac{i}{\sqrt{\hbar}}\left(s-\left(P+\frac{\sqrt{\hbar} p}{2}\right) q\right)\right) K_{S, P, Q}^{\hbar} \exp \left(i p \xi-q \frac{\partial}{\partial \xi}\right) f .
\end{gathered}
$$

В данной работе исследуются свойства оператора $K$, удовлетворяюшего соотношениям (6) и (7). Показывается, что и в общем случае числовой множитель $\chi_{y}(\delta y)$ не является произвольным, а выражается через $\delta y$ следующим образом: $\chi_{y}(\delta y)=e^{-i \omega(\delta y)}$, где величина $\omega(\delta y)$ линейно зависит от $\delta y$, т.е. является значением дифференциальной 1-формы $\omega$ на касательном векторе $\delta y$ к многообразию У в точке $y$. Аналогично, оператор $V_{y}[\delta y]$ выражается через оператор $\Omega(\delta y)$, линейно зависящий от $\delta y: V_{y}(\delta y)=e^{i \Omega(\delta y)}$. Для операторов $\Omega\left(\delta y_{1}\right)$ и $\Omega\left(\delta y_{2}\right)$ устанавливаются коммутационные соотношения:

$$
\left[\Omega\left(\delta y_{1}\right), \Omega\left(\delta y_{2}\right)\right]=\frac{1}{i} \omega\left(\delta y_{1}, \delta y_{2}\right)
$$

Через $d \omega$ обозначен дифференциал 1-формы $\omega$, являющийся дифференциальной 2-формой, а через $d \omega\left(\delta y_{1}, \delta y_{2}\right)$ значение этой 2-формы на паре касательных векторов $\delta y_{1}, \delta y_{2}$. 
Рассматриваются также примеры операторов, удовлетворяюших свойствам 1, 2 и отвечающих им 1-форм $\omega$ и $\Omega$. Для произвольного абстрактного канонического оператора вводится понятие фазового пространства, которое в частном случае (5) совпадает c $(2 n)$-мерньп линейным пространством с симплектической структурой $d P \wedge d Q$. Приводятся примеры, в которых фазовое пространство нетривиально (является тором, сферой, плоскостью Лобачевского, проективным пространством). Исследован вопрос об условиях квантования.

Часто встречаются случаи, когда пространство $\mathscr{F}$, которому принадлежит аргумент оператора $K_{y}^{\varepsilon}$, зависит от $y$. Поэтому вместо совокупности многообразия У и гильбертова пространства $\mathscr{F}$ следует в данном случае рассмотреть расслоение $\pi: \mathscr{Z} \rightarrow \mathscr{Y}$, базой которого является многообразие $\mathscr{Y}$, а слоем $\pi^{-1} y$ - зависящее от $y$ гильбертово пространство $\mathscr{F} y$. В частном случае, когда $\mathscr{F} y$ не зависит от $y$, данное расслоение является тривиальньм, т.е. пространство этого расслоения представляет собой прямое произведение $\mathscr{Z}=\mathscr{Y} \times \mathscr{F}$, а отображение $\pi$ выделяет из элемента данного пространства $(y, f)$ его $y$-компоненту.

Поскольку расслоение $\pi: \mathscr{Z} \rightarrow \mathscr{Y}$ используется при построении квазиклассических асимптотик в квантовой механике, будем назьвать это расслоение квазиклассическим, а его базу У - расширенным фазовым пространством. В частном случае (5) УУ представляет собой $(2 n+1)$-мерное пространство, состоящее из совокупностей $(S, P, Q)$ числа $S$ и $n$-мерных векторов $P, Q$.

Поскольку формула (2) является одной из возможных записей канонического оператора Маслова, отвечающего комплексному ростку в точке, будем назьвать оператор, входящий в формулу (4), абстрактным каноническим оператором. Приведем определение абстрактного канонического оператора, являющееся более общим, чем рассмотренное в [6].

2. Определение абстрактного канонического оператора. Будем говорить, что задан абстрактньй канонический оператор, если выполнены следующие условия.

I. Задано векторное (локально-тривиальное) расслоение $\pi: \mathscr{Z} \rightarrow \mathscr{Y}$, являющееся совокупностью топологического пространства $\mathscr{Z}$, гладкого многообразия $\mathscr{Y}$ и непрерьвного отображения $\pi$. На каждом слое расслоения $\pi^{-1} y \in \mathscr{Z}$ определены операции суммирования, умножения на число и скалярного произведения, непрерьвные в топологии пространства $\mathscr{Z}$, превращающие $\pi^{-1} y$ в евклидово пространство, состоящее более чем из одного нулевого элемента.

II. При каждом значении параметра $\varepsilon$, пробегающего некоторое подмножество $\{\varepsilon\}$ числовой прямой, содержащее 0 в качестве предельной точки, заданы гильбертовы пространства $\mathscr{H}^{\varepsilon}$.

III. Задана совокупность отображений $K^{\varepsilon}: \mathscr{Z} \rightarrow \mathscr{H}^{\varepsilon}$, удовлетворяющая следующим аксиомам.

Аксиома 1 (послойная линейность). При $z_{1}, z_{2} \in \pi^{-1} y, \alpha \in \mathbb{C}$

$$
K^{\varepsilon}\left(z_{1}+\alpha z_{2}\right)=K^{\varepsilon} z_{1}+\alpha K^{\varepsilon} z_{2}
$$

АКСИома 2. Для любого вещественного s определено послойное отображсние $g_{s}: \mathscr{Z} \rightarrow \mathscr{Z}$ makoe, чmo

$$
\left\|K^{\varepsilon} g_{\sqrt{\varepsilon} s_{1}+\varepsilon s_{2}} z-e^{i s_{1} / \sqrt{\varepsilon}} e^{i s_{2}} K^{\varepsilon} z\right\| \rightarrow 0, \quad \varepsilon \rightarrow 0 .
$$


Аксиома 3. Для любой непрерывной кривой $z(\tau)$ на топологическом пространстве $\mathscr{Z}$, имеющей гладкую проекиию $\pi z(\tau)$ на базу У, справедливо соотношение

$$
\left\|K^{\varepsilon} z(\sqrt{\varepsilon})\right\| \rightarrow\|z(0)\|, \quad \varepsilon \rightarrow 0 .
$$

В следующих трех аксиомах $\Phi$ - произвольное непрерьвное отображение $\Phi: \mathbb{R}^{k} \rightarrow \mathscr{Z}$, для которого отображение $\pi \Phi: \mathbb{R}^{k} \rightarrow \mathscr{Y}$ является гладким.

Аксиома 4. Для любых функиий $\xi_{1}(\tau)$ и $\xi_{2}(\tau): \mathbb{R} \rightarrow \mathbb{R}^{k} \operatorname{ma\kappa ux,~ито~} \xi_{1}(\tau)-\xi_{2}(\tau)=$ $O\left(\tau^{3}\right)$, справедливо соотношение

$$
\left\|K^{\varepsilon} \Phi\left(\xi_{1}(\sqrt{\varepsilon})\right)-K^{\varepsilon} \Phi\left(\xi_{2}(\sqrt{\varepsilon})\right)\right\| \rightarrow 0, \quad \varepsilon \rightarrow 0
$$

АкСиома 5. Для любой функиии $\xi: \mathbb{R} \rightarrow \mathbb{R}^{k}$ и любого вектора $\beta \in \mathbb{R}^{k}$ справедливо соотношение

$$
\left\|K^{\varepsilon} \Phi(\xi(\sqrt{\varepsilon})+\varepsilon \beta)-\chi_{\xi(0), \pi \Phi}[\beta] K^{\varepsilon} \Phi(\xi(\sqrt{\varepsilon}))\right\| \rightarrow 0, \quad \varepsilon \rightarrow 0
$$

для некоторого комплексного мнохителя $\chi_{\xi(0), \pi \Phi}[\beta]$, гладко зависящего от $\beta$.

Аксиома 6. Для любой функиии $\xi: \mathbb{R} \rightarrow \mathbb{R}^{k}$ и любого вектора $\beta \in \mathbb{R}^{k}$ существуют линейные операторы $V_{\pi \Phi}(\xi, \beta)$, действующие в слое $\pi^{-1} \pi \Phi(\xi)$, сильно непрерывные по $\beta$, и комплексные числа $c_{\Phi}^{\varepsilon}(\xi, \beta)$ такие, что

$$
\left\|K^{\varepsilon} \Phi(\xi(\sqrt{\varepsilon})+\sqrt{\varepsilon} \beta)-c_{\Phi}^{\varepsilon}(\xi(\sqrt{\varepsilon}), \beta) K^{\varepsilon} V_{\pi \Phi}(\xi(\sqrt{\varepsilon}), \beta) \Phi(\xi(\sqrt{\varepsilon}))\right\| \rightarrow 0, \quad \varepsilon \rightarrow 0 .
$$

При $\chi_{\xi(0), \pi \Phi}[\beta]=1$ множитель $c_{\Phi}^{\varepsilon}$ не зависит от $\varepsilon$.

ЗАмЕчАниЕ. Аксиома 2 означает, что при некотором преобразовании $g_{s}$ абстрактный канонический оператор домножается на числовой множитель. Для оператора (5) преобразование $g_{s}$ сдвигает аргумент $S$ на $s$. Аксиомы 4-6 определяют преобразование абстрактного канонического оператора при сдвиге $z$ на величину $O\left(\varepsilon^{3 / 2}\right), O(\varepsilon)$ и $O\left(\varepsilon^{1 / 2}\right)$ соответственно.

\section{3. Свойства абстрактного канонического оператора.}

ЛЕмма 1. Комплексный множитель $\chi_{\xi, \pi \Phi}[\beta]$ однозначно определяется из свойcmвa (8).

ДокАЗАТЕЛЬСтвО. Пусть для двух множителей $\chi^{1}$ и $\chi^{2}$ выполнено свойство (8). Выбирая в качестве функции $\xi(\varepsilon)$ константу, имеем

$$
\begin{aligned}
& \left\|K^{\varepsilon} \Phi(\xi+\varepsilon \beta)-\chi_{\xi, \pi \Phi}^{1}[\beta] K^{\varepsilon} \Phi(\xi)\right\| \rightarrow 0, \\
& \left\|K^{\varepsilon} \Phi(\xi+\varepsilon \beta)-\chi_{\xi, \pi \Phi}^{2}[\beta] K^{\varepsilon} \Phi(\xi)\right\| \rightarrow 0,
\end{aligned}
$$

Величина $\left\|\chi_{\xi, \pi \Phi}^{1}[\beta] K^{\varepsilon} \Phi(\xi)-\chi_{\xi, \pi \Phi}^{2}[\beta] K^{\varepsilon} \Phi(\xi)\right\|$ в силу неравенства треугольника и (10) при $\varepsilon \rightarrow 0$ стремится к 0 , а в силу аксиомы 3 она стремится к $\left|\chi_{\xi, \pi \Phi}^{1}[\beta]-\chi_{\xi, \pi \Phi}^{2}[\beta]\right| \cdot\|\Phi(\xi)\|$. Так как для некоторого отображения $\Phi$, отвечающего данному отображению $\pi \Phi$, вектор $\Phi(\xi)$ ненулевой, то $\chi^{1}=\chi^{2}$ при любом $\pi \Phi$. Лемма 1 доказана. 
Лемма 2. Выполнены следующие свойства:

$$
\chi_{\xi, \pi \Phi}[0]=1, \quad\left|\chi_{\xi, \pi \Phi}[\beta]\right|=1, \quad \chi_{\xi, \pi \Phi}[\beta+\gamma]=\chi_{\xi, \pi \Phi}[\beta] \chi_{\xi, \pi \Phi}[\gamma] .
$$

ДОКАЗАТЕЛЬСТВО. При $\beta=0$ свойство (8) выполнено, если $\chi_{\xi, \pi \Phi}[0]=1$. Положим $Y^{\varepsilon}=K^{\varepsilon} \Phi(\xi+\varepsilon \beta)-\chi_{\xi, \pi \Phi}[\beta] K^{\varepsilon} \Phi(\xi)$. Из неравенства треугольника вытекает, что

$$
\left\|\chi_{\xi, \pi \Phi}[\beta] K^{\varepsilon} \Phi(\xi)\right\|-\left\|Y^{\varepsilon}\right\| \leqslant\left\|K^{\varepsilon} \Phi(\xi+\varepsilon \beta)\right\| \leqslant \chi_{\xi, \pi \Phi}[\beta] K^{\varepsilon} \Phi(\xi)+\left\|Y^{\varepsilon}\right\| .
$$

В силу аксиомы $3\left\|K^{\varepsilon} \Phi(\xi)\right\| \rightarrow\|\Phi(\xi)\|$ при $\varepsilon \rightarrow 0$, а в силу аксиомы $5\left\|Y^{\varepsilon}\right\| \rightarrow 0$ при $\varepsilon \rightarrow 0$. Поэтому

$$
\left\|K^{\varepsilon} \Phi(\xi+\varepsilon \beta)\right\| \rightarrow\left|\chi_{\xi, \pi \Phi}[\beta]\right| \cdot\|\Phi(\xi)\|, \quad \varepsilon \rightarrow 0 .
$$

С другой стороны, из аксиомы 3 вытекает, что

$$
\left\|K^{\varepsilon} \Phi(\xi+\varepsilon \beta)\right\| \rightarrow\|\Phi(\xi)\|, \quad \varepsilon \rightarrow 0 .
$$

Отсюда $\left|\chi_{\xi, \pi \Phi}[\beta]\right|=1$.

Выберем в качестве функции $\xi(\sqrt{\varepsilon})$ функцию вида $\xi(\sqrt{(} \varepsilon))=\xi+\varepsilon \gamma$. Из формулы (8) вытекает, что

$$
\begin{gathered}
\left\|K^{\varepsilon} \Phi(\xi+\varepsilon \gamma+\varepsilon \beta)-\chi_{\xi, \pi \Phi}[\beta] K^{\varepsilon} \Phi(\xi+\varepsilon \gamma)\right\| \rightarrow 0, \\
\left\|\chi_{\xi, \pi \Phi}[\beta] K^{\varepsilon} \Phi(\xi+\varepsilon \gamma)-\chi_{\xi, \pi \Phi}[\beta] \chi_{\xi, \pi \Phi}[\gamma] K^{\varepsilon} \Phi(\xi)\right\| \rightarrow 0, \quad \varepsilon \rightarrow 0 .
\end{gathered}
$$

Складывая данные соотношения и применяя неравенство треугольника, получаем

$$
\left\|K^{\varepsilon} \Phi(\xi+\varepsilon \gamma+\varepsilon \beta)-\chi_{\xi, \pi \Phi}[\beta] \chi_{\xi, \pi \Phi}[\gamma] K^{\varepsilon} \Phi(\xi)\right\| \rightarrow 0, \quad \varepsilon \rightarrow 0 .
$$

В силу однозначности определения множителя $\chi$ из свойства (8) получаем третье утверждение леммы. Лемма 2 доказана.

СлЕДСТВИЕ. Функиия $\chi_{\xi, \pi \Phi}[\beta]$ представляется в виде

$$
\chi_{\xi, \pi \Phi}[\beta]=\exp \left(-i \sum_{l=1}^{k} \omega_{l}(\xi, \Phi) \beta_{l}\right),
$$

где $\omega_{l}(\xi, \Phi)$ - некоторые вещественные коэффициенты.

Для исследования свойств оператора $V_{\pi \Phi}$ докажем некоторые вспомогательные утверждения.

Лемма 3. Пусть $f, g \in \pi^{-1} y$. Тогда $\left(K^{\varepsilon} f, K^{\varepsilon} g\right) \rightarrow(f, g) n p u \varepsilon \rightarrow 0$.

ДоКАЗАТЕЛЬСТво. В силу аксиомы 3 имеем

$$
\begin{aligned}
2 \operatorname{Re}\left(K^{\varepsilon} f, K^{\varepsilon} g\right) & =\left(K^{\varepsilon}(f+g), K^{\varepsilon}(f+g)\right)-\left(K^{\varepsilon} f, K^{\varepsilon} f\right)-\left(K^{\varepsilon} g, K^{\varepsilon} g\right) \\
& \rightarrow(f+g, f+g)-(f, f)-(g, g)=2 \operatorname{Re}(f, g), \\
-2 \operatorname{Im}\left(K^{\varepsilon} f, K^{\varepsilon} g\right) & =\left(K^{\varepsilon}(f+i g), K^{\varepsilon}(f+i g)\right)-\left(K^{\varepsilon} f, K^{\varepsilon} f\right)-\left(K^{\varepsilon} g, K^{\varepsilon} g\right) \\
& \rightarrow(f+i g, f+i g)-(f, f)-(g, g)=-2 \operatorname{Im}(f, g)
\end{aligned}
$$

при $\varepsilon \rightarrow 0$. Лемма 3 доказана. 
Лемма 4. Пусть $f, g \in \pi^{-1} y, f \neq 0, u$ для некоторого зависящего от $\varepsilon$ комплексного множителя $\alpha^{\varepsilon}$

$$
\left\|K^{\varepsilon} f-\alpha^{\varepsilon} K^{\varepsilon} g\right\| \rightarrow 0, \quad \varepsilon \rightarrow 0
$$

Тогда существует

$$
\lim _{\varepsilon \rightarrow 0} \alpha^{\varepsilon}=\alpha \neq 0
$$

$u f=\alpha g$.

ДокАЗАтЕльство. Так как $\left\|K^{\varepsilon} f\right\| \rightarrow\|f\| \neq 0$ при $\varepsilon \rightarrow 0$, то $g \neq 0$. Положим $y^{\varepsilon}=K^{\varepsilon} f-\alpha^{\varepsilon} K^{\varepsilon} g$. В силу неравенства треугольника

$$
\left\|K^{\varepsilon} f\right\|-\left\|y^{\varepsilon}\right\| \leqslant\left\|\alpha^{\varepsilon} K^{\varepsilon} g\right\| \leqslant\left\|K^{\varepsilon} f\right\|+\left\|y^{\varepsilon}\right\|,
$$

поэтому $\left|\alpha^{\varepsilon}\right| \cdot\left\|K^{\varepsilon} g\right\| \rightarrow\|f\|$ при $\varepsilon \rightarrow 0$. Так как $\left\|K^{\varepsilon} g\right\| \rightarrow\|g\|$ при $\varepsilon \rightarrow 0$, то $\left|\alpha^{\varepsilon}\right| \rightarrow\|f\| /\|g\|$ при $\varepsilon \rightarrow 0$. Далее, справедливо тождество

$$
2 \operatorname{Re}\left(\alpha^{\varepsilon}(f, g)\right)=-2 \operatorname{Re}\left(\alpha^{\varepsilon}\left(\left(K^{\varepsilon} f, K^{\varepsilon} g\right)-(f, g)\right)\right)-\left\|y^{\varepsilon}\right\|^{2}+\left(K^{\varepsilon} f, K^{\varepsilon} f\right)+\left|\alpha^{\varepsilon}\right|^{2}\left(K^{\varepsilon} g, K^{\varepsilon} g\right) \text {. }
$$

Первые два слагаемых в правой части этого равенства стремятся к 0 , а остальные два слагаемых стремятся к $\|f\|^{2}$. Поэтому

$$
\operatorname{Re}\left(\alpha^{\varepsilon}(f, g)\right) \rightarrow\|f\|^{2}, \quad \varepsilon \rightarrow 0 .
$$

Далее,

$$
\left|\alpha^{\varepsilon}(f, g)\right|^{2} \rightarrow \frac{\|f\|^{2}}{\|g\|^{2}}|(f, g)|^{2}, \quad \varepsilon \rightarrow 0
$$

Поэтому

$$
\left(\operatorname{Im}\left(\alpha^{\varepsilon}(f, g)\right)\right)^{2} \rightarrow \frac{\|f\|^{2}}{\|g\|^{2}}\left(|(f, g)|^{2}-\|f\|^{2} \cdot\|g\|^{2}\right), \quad \varepsilon \rightarrow 0 .
$$

Левая часть формулы (13) неотрицательна, а правая часть в силу неравенства КошиБуняковского неположительна. Поэтому

$$
\left(\operatorname{Im}\left(\alpha^{\varepsilon}(f, g)\right)\right)^{2} \rightarrow 0, \quad \varepsilon \rightarrow 0 .
$$

В силу (12) получаем, что $\alpha^{\varepsilon} \rightarrow\|f\|$ при $\varepsilon \rightarrow 0$, откуда $\alpha^{\varepsilon} \rightarrow \alpha=\|f\| /(f, g)$ при $\varepsilon \rightarrow 0$. Следовательно, $\left\|\left(\alpha^{\varepsilon}-\alpha\right) K^{\varepsilon} g\right\| \rightarrow 0$ при $\varepsilon \rightarrow 0$ и в силу (11) по неравенству треугольника $\left\|K^{\varepsilon}(f-\alpha g)\right\| \rightarrow 0$ при $\varepsilon \rightarrow 0$. Из аксиомы 3 сразу же вытекает $f=\alpha g$. Лемма 4 доказана.

Оказьвается, что зависящий от $\varepsilon$ числовой коэффициент $c^{\varepsilon}(\xi, \beta)$ определяется неоднозначно, а с точностью до умножения на регулярньй при $\varepsilon \rightarrow 0$ множитель.

ЛЕмма 5. Пусть для коэффициента $\widetilde{c}_{\Phi}^{\varepsilon}(\xi, \beta)$ и оператора $\widetilde{V}_{\pi \Phi}(\xi, \beta)$ также выполнено свойство (9). Тогда

$$
\frac{\widetilde{c}_{\Phi}^{\varepsilon}(\xi, \beta)}{c_{\Phi}^{\varepsilon}(\xi, \beta)} \rightarrow \lambda_{\pi \Phi}(\xi, \beta) \neq 0, \quad \varepsilon \rightarrow 0, \quad \widetilde{V}_{\pi \Phi}(\xi, \beta)=\frac{1}{\lambda_{\pi \Phi}(\xi, \beta)} V_{\pi \Phi}(\xi, \beta)
$$


ДокАЗАТЕльСтво. В силу свойства $\left\|K^{\varepsilon} \Phi(\xi+\sqrt{\varepsilon} \beta)\right\| \rightarrow\|\Phi(\xi)\| \neq 0$ при $\varepsilon \rightarrow 0$ коэффициенты $\widetilde{c}_{\Phi}^{\varepsilon}(\xi, \beta)$ и $c_{\Phi}^{\varepsilon}(\xi, \beta)$ отличны от 0 для достаточно малых $\varepsilon$. Из (9) вытекает, что

$$
\left|c_{\Phi}^{\varepsilon}(\xi, \beta)\right| \cdot\left\|K^{\varepsilon} V_{\pi \Phi}(\xi, \beta) \Phi(\xi)\right\| \rightarrow\|\Phi(\xi)\|, \quad \varepsilon \rightarrow 0,
$$

а из аксиомы 3 следует, что

$$
\left\|K^{\varepsilon} V_{\pi \Phi}(\xi, \beta) \Phi(\xi)\right\| \rightarrow\left\|V_{\pi \Phi}(\xi, \beta) \Phi(\xi)\right\|, \quad \varepsilon \rightarrow 0 .
$$

Отсюда получаем

$$
\left|c_{\Phi}^{\varepsilon}(\xi, \beta)\right|^{-1} \rightarrow \frac{\left\|V_{\pi \Phi}(\xi, \beta) \Phi(\xi)\right\|}{\|\Phi(\xi)\|}, \quad \varepsilon \rightarrow 0 .
$$

Из свойства (9), неравенства треугольника и формулы (14) вытекает, что

$$
\left\|K^{\varepsilon} V_{\pi \Phi}(\xi, \beta) \Phi(\xi)-\frac{\widetilde{c}_{\Phi}^{\varepsilon}(\xi, \beta)}{c_{\Phi}^{\varepsilon}(\xi, \beta)} K^{\varepsilon} \widetilde{V}_{\pi \Phi}(\xi, \beta) \Phi(\xi)\right\| \rightarrow 0, \quad \varepsilon \rightarrow 0 .
$$

Применяя лемму 4, получаем

$$
\frac{\widetilde{c}_{\Phi}^{\varepsilon}(\xi, \beta)}{c_{\Phi}^{\varepsilon}(\xi, \beta)} \rightarrow \lambda_{\pi \Phi}(\xi, \beta), \quad \varepsilon \rightarrow 0, \quad V_{\pi \Phi}(\xi, \beta) \Phi(\xi)=\lambda_{\pi \Phi}(\xi, \beta) \widetilde{V}_{\pi \Phi}(\xi, \beta) \Phi(\xi) .
$$

Лемма 5 доказана.

ЛЕмма 6. Числовой множитель $c_{\Phi}^{\varepsilon}(\xi, \beta)$, входящий в формулу (9), представляется в виде

$$
c_{\Phi}^{\varepsilon}(\xi, \beta)=\exp \left(-\frac{i}{\sqrt{\varepsilon}} \sum_{l=1}^{k} \omega_{l}(\xi, \Phi) \beta_{l}\right) \lambda_{\pi \Phi}(\xi, \beta)
$$

для некоторого не зависящего от $\varepsilon$ коэффициента $\lambda_{\pi \Phi}(\xi, \beta) \neq 0$.

ДоказАтельство. Рассмотрим отображение $F: \mathbb{R}^{k+1} \rightarrow \mathscr{Z}$ вида $F(\xi, s)=g_{s} \Phi(\xi)$, $\xi \in \mathbb{R}^{k}, s \in \mathbb{R}$. Записьвая для этого отображения аксиому 6 , получаем

$$
\left\|K^{\varepsilon} F(\xi+\sqrt{\varepsilon} \beta, \sqrt{\varepsilon} s)-c_{F}^{\varepsilon}(\xi, 0 ; \beta, s) K^{\varepsilon} V_{\pi F}(\xi, 0 ; \beta, s) F(\xi, 0)\right\| \rightarrow 0, \quad \varepsilon \rightarrow 0 .
$$

Докажем, что при

$$
s(\beta)=\sum_{l=1}^{k} \omega_{l}(\xi, \Phi) \beta_{l}
$$

выполнено соотношение

$$
\chi_{\xi, 0, \pi F}[\beta, s(\beta)]=1,
$$

т.е. множитель $c_{F}^{\varepsilon}(\xi, 0 ; \beta, s)$ не зависит от $\varepsilon$ :

$$
\lambda_{\pi \Phi}(\xi, \beta)=c_{F}^{\varepsilon}(\xi, 0 ; \beta, s) .
$$

Из аксиомы 2 следует, что

$$
\left\|K^{\varepsilon} F(\xi+\varepsilon \beta, \varepsilon s)-e^{i s} K^{\varepsilon} F(\xi+\varepsilon \beta, 0)\right\| \rightarrow 0, \quad \varepsilon \rightarrow 0 .
$$


Из аксиомы 5 и следствия из леммы 2 имеем

$$
\left\|e^{i s} K^{\varepsilon} F(\xi+\varepsilon \beta, 0)-\exp \left(i\left(s-\sum_{l=1}^{k} \omega_{l}(\xi, \Phi) \beta_{l}\right)\right) K^{\varepsilon} F(\xi, 0)\right\| \rightarrow 0, \quad \varepsilon \rightarrow 0
$$

Учитьвая (16), получаем $\left\|K^{\varepsilon} F(\xi+\varepsilon \beta, \varepsilon s)-K^{\varepsilon} F(\xi, 0)\right\| \rightarrow 0$ при $\varepsilon \rightarrow 0$. Соотношения (17) и (18) доказаны.

Из аксиомы 2 получаем

$$
\left\|K^{\varepsilon} F(\xi+\sqrt{\varepsilon} \beta, \sqrt{\varepsilon} s)-e^{i s / \sqrt{\varepsilon}} K^{\varepsilon} F(\xi+\sqrt{\varepsilon} \beta, 0)\right\| \rightarrow 0, \quad \varepsilon \rightarrow 0 .
$$

Учитьвая равенство $\Phi(\xi)=F(\xi, 0)$ и соотношение $(15)$, а также неравенство треугольника, имеем

$$
\left\|e^{i s / \sqrt{\varepsilon}} K^{\varepsilon} \Phi(\xi+\sqrt{\varepsilon} \beta)-\lambda_{\pi \Phi}(\xi, \beta) K^{\varepsilon} V_{\pi F}(\xi, 0 ; \beta, s(\beta)) \Phi(\xi)\right\| \rightarrow 0, \quad \varepsilon \rightarrow 0 .
$$

Сравнивая данное соотношение с аксиомой 6 и учитьвая (16), получаем утверждение леммы 6.

Исследуем свойства операторов $V_{\pi \Phi}(\xi, \beta)$. В силу леммы 5 эти операторы определены с точностью до числового множителя. Зафиксируем процедуру выбора этого множителя.

ЛЕмма 7. Существует однозначно определенный числовой множстель $\rho_{\xi, \Phi}[\beta]$, отличный от 0, такой, что операторы $U_{\xi, \pi \Phi}(\beta)=\rho_{\xi, \Phi}[\beta] V_{\pi \Phi}[\xi, \beta]$ удовлетворяют свойствам:

1) операторы $U_{\xi, \pi \Phi}(\beta)$ унитарны;

2) $U_{\xi, \pi \Phi}(0)=1$;

3) выполнено

$$
U_{\xi, \pi \Phi}(\beta+\gamma)=\exp \left(-\frac{i}{2} \sum_{l, s=1}^{k}\left(\frac{\partial \omega_{l}}{\partial \xi_{s}}-\frac{\partial \omega_{s}}{\partial \xi_{l}}\right) \gamma_{s} \beta_{l}\right) U_{\xi, \pi \Phi}(\gamma) U_{\xi, \pi \Phi}(\beta)
$$

ДокАЗАТЕЛЬСТво. Обозначим через $\widetilde{V}_{x i, \pi \Phi}$ однозначно определенные операторы вида

$$
\widetilde{V}_{\xi, \pi \Phi}=\lambda_{\pi \Phi}(\xi, \beta) V_{\pi \Phi}(\xi, \beta),
$$

где множитель $\lambda_{\pi \Phi}$ определен в лемме 6 . Из аксиомы 6 вытекает, что

$$
\left\|K^{\varepsilon} \Phi(\xi+\sqrt{\varepsilon} \beta)-\exp \left(-\frac{i}{\sqrt{\varepsilon}} \sum_{l=1}^{k} \omega_{l}(\xi, \Phi) \beta_{l}\right) K^{\varepsilon} \widetilde{V}_{\xi, \pi \Phi}(\beta) \Phi(\xi)\right\| \rightarrow 0, \quad \varepsilon \rightarrow 0
$$

Так как $\left\|K^{\varepsilon} \Phi(\xi+\sqrt{\varepsilon} \beta)\right\| \rightarrow\|\Phi(\xi)\|$ при $\varepsilon \rightarrow 0$, то и предел нормы $\left\|K^{\varepsilon} \widetilde{V}_{\xi, \pi \Phi}(\beta) \Phi(\xi)\right\|$ при $\varepsilon \rightarrow 0$ равен $\|\Phi(\xi)\|$. С другой стороны, по аксиоме 3 этот предел равен $\left\|\widetilde{V}_{\xi, \pi \Phi}(\beta) \Phi(\xi)\right\|$. 
Поэтому операторы $\widetilde{V}_{\xi, \pi \Phi}(\beta)$ изометричны. Подставляя в формулу (19) $\beta=0$, получаем, что $\widetilde{V}_{\xi, \pi \Phi}(0)=1$. Применяя формулу $(9)$ для $\xi(\sqrt{\varepsilon})=\xi+\sqrt{\varepsilon} \gamma$, получаем

$$
\begin{aligned}
\| K^{\varepsilon} \Phi(\xi+\sqrt{\varepsilon} \beta+\sqrt{\varepsilon} \gamma)- & \exp \left(-\frac{i}{\sqrt{\varepsilon}} \sum_{l=1}^{k} \omega_{l}(\xi+\sqrt{\varepsilon} \gamma, \Phi) \beta_{l}\right) \\
\times & \times K^{\varepsilon} \widetilde{V}_{\xi+\sqrt{\varepsilon} \gamma, \pi \Phi}(\beta) \Phi(\xi+\sqrt{\varepsilon} \gamma) \| \rightarrow 0, \quad \varepsilon \rightarrow 0 .
\end{aligned}
$$

Далее,

$$
\begin{aligned}
\| K^{\varepsilon} \widetilde{V}_{\xi+\sqrt{\varepsilon} \gamma, \pi \Phi}(\beta) \Phi(\xi+\sqrt{\varepsilon} \gamma)- & \exp \left(-\frac{i}{\sqrt{\varepsilon}} \sum_{l=1}^{k} \omega_{l}(\xi, \Phi) \gamma_{l}\right) \\
& \times K^{\varepsilon} \widetilde{V}_{\xi, \pi \Phi}(\gamma) \widetilde{V}_{\xi, \pi \Phi}(\beta) \Phi(\xi) \| \rightarrow 0, \quad \varepsilon \rightarrow 0 .
\end{aligned}
$$

Из неравенства треугольника следует, что

$$
\begin{aligned}
\| K^{\varepsilon} \Phi(\xi+\sqrt{\varepsilon} \beta+\sqrt{\varepsilon} \gamma)- & \exp \left(-\frac{i}{\sqrt{\varepsilon}} \sum_{l=1}^{k}\left(\omega_{l}(\xi+\sqrt{\varepsilon} \gamma, \Phi) \beta_{l} \omega_{l}(\xi, \Phi) \gamma_{l}\right)\right) \\
& \times K^{\varepsilon} \widetilde{V}_{\xi, \pi \Phi}(\gamma) \widetilde{V}_{\xi, \pi \Phi}(\beta) \Phi(\xi) \| \rightarrow 0, \quad \varepsilon \rightarrow 0 .
\end{aligned}
$$

С другой стороны, формула (9) дает

$$
\left\|K^{\varepsilon} \Phi(\xi+\sqrt{\varepsilon}(\beta+\gamma))-\exp \left(-\frac{i}{\sqrt{\varepsilon}} \sum_{l=1}^{k} \omega_{l}(\xi, \Phi)\left(\beta_{l}+\gamma_{l}\right)\right) K^{\varepsilon} \widetilde{V}_{\xi, \pi \Phi}(\beta+\gamma) \Phi(\xi)\right\| \rightarrow 0, \quad \varepsilon \rightarrow 0 .
$$

Поэтому

$$
\begin{aligned}
\| K^{\varepsilon} \widetilde{V}_{\xi, \pi \Phi}(\beta+\gamma) \Phi(\xi)- & \exp \left(-\frac{i}{\sqrt{\varepsilon}} \sum_{l=1}^{k}\left(\omega_{l}(\xi+\sqrt{\varepsilon} \gamma, \Phi)-\omega_{l}(\xi, \Phi)\right) \beta_{l}\right) \\
& \times K^{\varepsilon} \widetilde{V}_{\xi, \pi \Phi}(\gamma) \widetilde{V}_{\xi, \pi \Phi}(\beta) \Phi(\xi) \| \rightarrow 0, \quad \varepsilon \rightarrow 0 .
\end{aligned}
$$

Отсюда

$$
\widetilde{V}_{\xi, \pi \Phi}(\beta+\gamma)=\exp \left(-i \frac{\partial \omega_{l}}{\partial \xi_{s}} \gamma_{s} \beta_{l}\right) \widetilde{V}_{\xi, \pi \Phi}(\gamma) \widetilde{V}_{\xi, \pi \Phi}(\beta) .
$$

Переобозначая

$$
U_{\xi, \pi \Phi}(\beta)=\exp \left(i \frac{\partial \omega_{l}}{\partial \xi_{s}}(\xi, \Phi) \beta_{s} \beta_{l}\right) \widetilde{V}_{\xi, \pi \Phi}(\beta),
$$

получаем формулу (18). Унитарность операторов $U_{\xi, \pi \Phi}(\beta)$ следует из изометричности операторов $\widetilde{V}_{\xi, \pi \Phi}(\beta)$ и обратимости операторов $U_{\xi, \pi \Phi}(\beta)$, вытекающей из свойства $(18)$ при $\beta=-\gamma$. Лемма 7 доказана.

Рассмотрим пополнение $\left[\pi^{-1} y\right]$ евклидова пространства $\pi^{-1} y$, являющееся гильбертовым пространством. В силу ограниченности построенные операторы $U_{\xi, \pi \Phi}$ продолжаются на пространство $\left[\pi^{-1} y\right]$.

Аналогично теореме Стоуна доказывается 
СлЕДСТВИЕ. Onератор $U_{\xi, \pi \Phi}(\beta)$ представляется в виде

$$
U_{\xi, \pi \Phi}(\beta)=\exp \left(i \sum_{l=1}^{k} \Omega_{l}(\xi, \Phi) \beta_{l}\right),
$$

әде самосопряжсенные операторы $\Omega_{l}$, действующие в $\left[\pi^{-1} y\right]$, удовлетворяют коммутационным соотношениям

$$
\left[\Omega_{s}, \Omega_{l}\right]=i\left(\frac{\partial \omega_{s}}{\partial \xi_{l}}-\frac{\partial \omega_{l}}{\partial \xi_{s}}\right)
$$

на некоторой плотной области определения.

Из доказанных утверждений вытекает следующая теорема.

Tеорема 1. Пусть $\xi(\tau)=\xi+\alpha \tau+\beta \tau^{2}+O\left(\tau^{3}\right)$. Тогда

$$
\begin{aligned}
& \| K^{\varepsilon} \Phi(\xi(\sqrt{\varepsilon}))-\exp \left(-\frac{i}{\sqrt{\varepsilon}} \omega_{l}(\xi, \Phi)\left(\alpha_{l}+\beta_{l} \sqrt{\varepsilon}\right)\right) \exp \left(-\frac{i}{2} \frac{\partial \omega_{l}}{\partial \xi_{s}}(\xi, \Phi) \alpha_{l} \alpha_{s}\right) \\
& \times K^{\varepsilon} e^{i \Omega_{l}(\xi, \Phi) \alpha_{l}} \Phi(\xi) \| \rightarrow 0, \quad \varepsilon \rightarrow 0 .
\end{aligned}
$$

Исследуем зависимость чисел $\omega_{l}(\xi, \Phi)$ и операторов $\Omega_{l}(\xi, \Phi)$ от отображения $\Phi$.

Пусть $y(\tau)$ - гладкая кривая на базе $\mathscr{Y ~ , ~ я в л я ю щ е и ̆ с я ~ г л а д к и м ~ м н о г о о б р а з и е м . ~ Ч е р е з ~}$ $d y / d \tau$ будем обозначать касательный вектор к данной кривой в точке $\tau$. Аналогично, сопоставим отображению $y=y\left(\xi_{1}, \ldots, \xi_{k}\right)$ набор векторов $\partial y / \partial \xi_{l}, l=\overline{1, k}$, принадлежащих касательному пространству к многообразию Я в точке $y(\xi)$.

Теорема 2. 1. Существует дифференциальная 1-форма $\omega$ на многообразии У такая, что ее значение на касательном векторе $\partial(\pi \Phi) / \partial \xi_{l}$ в точке $\pi \Phi(\xi)$ равно $\omega_{l}(\xi, \Phi)$.

2. Существует операторнозначная дифференциальная 1-форма $\Omega$ на многообразии У такая, что ее значение на касательном векторе $\partial(\pi \Phi) / \partial \xi_{l}$ в точке $\pi \Phi(\xi)$ равно $\Omega_{l}(\xi, \Phi)$.

ЗАмЕчАниЕ. Под операторнозначной 1-формой на У понимается совокупность линейных отображений $\Omega_{y}$ из касательного пространства $T \mathscr{Y}_{y}$ в пространство операторов, действующих в $\left[\pi^{-1} y\right]$.

ДокАЗАТЕЛЬСТвО. В силу локальной тривиальности расслоения $\pi: \mathscr{Z} \rightarrow \mathscr{Y}$ для любого касательного вектора $\zeta$ к многообразию $\mathscr{Y ~ в ~ т о ч к е ~} y$ существует кривая $\Phi(\tau)$ на $\mathscr{Z}$ такая, что $\pi \Phi(0)=y$ и $d(\pi \Phi(0)) / d \tau=\zeta$. Сопоставим касательному вектору $\zeta$ число $\omega_{1}(0, \Phi)$ и оператор $\Omega_{1}(0, \Phi)$. Покажем, что данные отображения $\omega: \zeta \mapsto \omega_{1}(0, \Phi)$ и $\Omega: \zeta \mapsto \Omega_{1}(0, \Phi)$ не зависят от выбора кривой $\pi \Phi$ и определяют 1 -форму $\omega_{1}$ и операторнозначную 1-форму $\Omega$.

Для доказательства однозначности построенных отображений воспользуемся локальной тривиальностью расслоения $\pi: \mathscr{Z} \rightarrow \mathscr{Y}:$ существует окрестность $O_{y}$ точки $y$, диффеоморфная пространству $\mathbb{R}^{n}$ (или гильбертову пространству $\mathscr{H}$ в случае бесконечномерного многообразия $\mathscr{Y})$, такая, что $\pi^{-1} O_{y}=O_{y} \times \mathscr{F}$, где $\mathscr{F}-$ гильбертово пространство. Таким образом, кривой $\Phi(\tau)$ при $\tau \in\left(0, \tau_{0}\right)$ сопоставляется упорядоченная пара $(y(\tau), f(\tau)), y(\tau) \in O_{y}, f(\tau) \in \mathscr{F}$, причем $\pi \Phi(\tau)=y(\tau)$. Однозначность отображений вытекает из следующей леммы. 
Лемма 8. Пусть $\Phi(\tau)=(y(\tau), f(\tau)), \widetilde{\Phi}(\tau)=(\widetilde{y}(\tau))-$ две кривые на $O_{y} \times \mathscr{F}$ такие, что

$$
y(0)=\widetilde{y}(0), \quad \frac{d y}{d \tau}(0)=\frac{d \widetilde{y}}{d \tau}(0) .
$$

Тогда $\omega_{1}(0, \Phi)=\omega_{1}(0, \widetilde{\Phi})$ u $\Omega_{1}(0, \Phi)=\Omega_{1}(0, \widetilde{\Phi})$.

ДокаЗАтельство. Рассмотрим отображение $\phi: \mathbb{R}^{2} \rightarrow O_{y} \times \mathscr{F}$ вида

$$
\phi(\tau, \alpha)=\left(y(\tau)+\frac{\widetilde{y}(\tau)-y(\tau)}{\tau^{2}} \alpha, f(\tau)\right),
$$

связанное с $\Phi$ и $\widetilde{\Phi}: \phi(\tau, 0)=\Phi(\tau), \phi\left(\tau, \tau^{2}\right)=\widetilde{\Phi}(\tau)$. Из теоремы 1 вытекает, что

$$
\begin{aligned}
\| K^{\varepsilon} \phi\left(\alpha \sqrt{\varepsilon}+\beta \varepsilon, \gamma \varepsilon+\rho \varepsilon^{2}\right)- & \exp \left(-\frac{i}{\sqrt{\varepsilon}} \omega_{1}(0, \phi)(\alpha+\beta \sqrt{\varepsilon})-i \omega_{2}(0, \phi) \gamma\right) \\
& \times \exp \left(-\frac{i}{2} \frac{\partial \omega_{1}}{\partial \xi_{1}}(\xi, \phi) \alpha^{2}\right) K^{\varepsilon} e^{i \Omega_{1}(\xi, \Phi)} \Phi(0) \| \rightarrow 0, \quad \varepsilon \rightarrow 0 .
\end{aligned}
$$

Подставляя в формулу (20) $\alpha=\gamma=\rho=0$ и сравнивая ее с формулой (19), в силу однозначности $\omega_{1}$ получаем $\omega_{1}(0, \Phi)=\omega_{1}(0, \phi)$. Аналогично, при $\alpha=\gamma=0, \rho=\beta^{2}$ имеем $\omega_{1}(0, \widetilde{\Phi})=\omega_{1}(0, \phi)$. При $\beta=\gamma=\rho=0$ получаем

$$
\frac{\partial \omega_{1}}{\partial \xi_{1}}(0, \Phi)=\frac{\partial \omega_{1}}{\partial \xi_{1}}(0, \phi), \quad \Omega_{1}(0, \Phi)=\Omega_{1}(0, \phi),
$$

а при $\beta=\rho=0, \gamma=\alpha^{2}$

$$
\frac{\partial \omega_{1}}{\partial \xi_{1}}(0, \Phi)=\frac{\partial \omega_{1}}{\partial \xi_{1}}(0, \phi)+2 \omega_{2}(0, \phi), \quad \Omega_{1}(0, \widetilde{\Phi})=\Omega_{1}(0, \phi) .
$$

Лемма 8 доказана.

Выражение $\Phi: \mathbb{R}^{k} \rightarrow \mathscr{Z}$, рассмотренное как функция одного из своих аргументов, является кривой на $\mathscr{Z}$, поэтому в силу леммы 8 значение $\omega$ на векторе $\partial(\pi \Phi) / \partial \xi_{l}$ в точке $\pi \Phi(\xi)$ равно $\omega_{l}(\xi, \Phi)$, а значение $\Omega$ на этом векторе равно $\Omega_{l}(\xi, \Phi)$.

Докажем, что отображения $\omega$ и $\Omega$ являются дифференциальными формами.

Лемма 9. Отображения $\omega$ и линейны.

ДокАЗАТЕЛЬСТво. Достаточно проверить, что для любых касательных к многообразию $\mathscr{Y}$ векторов $\zeta_{1}$ и $\zeta_{2}$ и $\lambda \in \mathbb{R}$ вьполнены соотношения

$$
\begin{aligned}
& \omega\left(\zeta_{1}+\lambda\left(\zeta_{2}-\zeta_{1}\right)\right)=(1-\lambda) \omega\left(\zeta_{1}\right)+\lambda \omega\left(\zeta_{2}\right), \\
& \Omega\left(\zeta_{1}+\lambda\left(\zeta_{2}-\zeta_{1}\right)\right)=(1-\lambda) \Omega\left(\zeta_{1}\right)+\lambda \Omega\left(\zeta_{2}\right) .
\end{aligned}
$$

Рассмотрим две кривые на $O_{y} \times \mathscr{F}:$

$$
\Phi_{0}(\tau)=\left(y_{0}(\tau), f(\tau)\right), \quad \Phi_{1}(\tau)=\left(y_{1}(\tau), f(\tau)\right),
$$


выходящие при $\tau=0$ из одной точки. Для доказательства формулы (21) проверим, что для отображения $\Phi_{\lambda}(\tau)=\left(y_{1}(\tau)+\lambda\left(y_{2}(\tau)-y_{1}(\tau)\right), f(\tau)\right)$ справедливы свойства

$$
\begin{aligned}
& \omega_{1}\left(0, \Phi_{\lambda}\right)=(1-\lambda) \omega_{1}\left(0, \Phi_{0}\right)+\lambda \omega_{1}\left(0, \Phi_{1}\right), \\
& \Omega_{1}\left(0, \Phi_{\lambda}\right)=(1-\lambda) \Omega_{1}\left(0, \Phi_{0}\right)+\lambda \Omega_{1}\left(0, \Phi_{1}\right) .
\end{aligned}
$$

Рассмотрим отображение

$$
\phi(\tau, \alpha)=\left(y_{1}(\tau)+\frac{y_{2}(\tau)-y_{1}(\tau)}{\tau} \alpha, f(\tau)\right)
$$

Поскольку $\Phi_{\lambda}(\tau)=\phi(\tau, \lambda \tau)$, из теоремы 1 по аналогии с доказательством леммы 8 получаем

$$
\omega_{1}\left(0, \Phi_{\lambda}\right)=\omega_{1}(0, \phi)+\lambda \omega_{2}(0, \phi), \quad \Omega_{1}\left(0, \Phi_{\lambda}\right)=\Omega_{1}(0, \phi)+\lambda \Omega_{2}(0, \phi) .
$$

Свойства (22), лемма 9 и теорема 2 доказаны.

4. Примеры абстрактных канонических операторов. В этом пункте приводятся примеры отображений, для которых выполнены аксиомы 1-6.

\section{1. Абстрактный канонический оператор и фазовое пространство.}

ПРИмеР 1. Рассмотрим тривиальное квазиклассическое расслоение с базой $\mathscr{Y}=$ $\mathbb{R}^{2 n+1}=\left\{(S, P, Q) \mid S \in \mathbb{R}, P \in \mathbb{R}^{n}, Q \in \mathbb{R}^{n}\right\}$ и слоем $L^{2}\left(\mathbb{R}^{n}\right)$. Пусть $\mathscr{H}^{\varepsilon}=L^{2}\left(\mathbb{R}^{n}\right)$. Рассмотрим абстрактньй канонический оператор $K^{\varepsilon}: \mathbb{R}^{2 n+1} \times L^{2}\left(\mathbb{R}^{n}\right) \rightarrow \mathscr{H}^{\varepsilon}$ вида

$$
\left(K^{\varepsilon}(S, P, Q ; f)\right)(x)=\frac{1}{\varepsilon^{n / 4}} \exp \left(\frac{i}{\varepsilon} S\right) \exp \left(\frac{i}{\varepsilon} P(x-Q)\right) f\left(\frac{x-Q}{\sqrt{\varepsilon}}\right)
$$

где $(S, P, Q) \in \mathscr{Y}, f \in L^{2}\left(\mathbb{R}^{n}\right)$. Ему отвечает 1-форма $\omega=-d S+P d Q$ и операторнозначная 1-форма $\Omega$, переводящая элемент $f$ пространства $\pi^{-1} y=L^{2}\left(\mathbb{R}^{n}\right)$ в дифференциальную 1-форму

$$
(\Omega f)(\xi)=d P \xi f(\xi)-d Q \frac{1}{i} \frac{\partial f}{\partial \xi} .
$$

Отображение $g_{s}$ является сдвигом $S$ на $s$.

В рассмотренном примере расширенное фазовое пространство Я является прямьг произведением пространства $\mathbb{R}$ и фазового пространства $\mathbb{R}^{2 n}$. Определим аналог понятия фазового пространства для произвольного абстрактного канонического оператора.

Рассмотрим действие преобразований $g_{s}$ на множестве У. Сопоставим каждому элементу этого множества $y \in \mathscr{Y}$ его орбиту $[y]=\left\{g_{s} y \mid s \in \mathbb{R}\right\}$. Множество всех таких орбит обозначим через $\mathscr{X}$. Будем назьвать множество $\mathscr{X}$ фазовым пространством.

Определим на $\mathscr{X}$ структуру гладкого многообразия, т.е. покажем, что локально $\mathscr{X}$ диффеоморфно $\mathbb{R}^{n-1}$ в случае $n$-мерного многообразия $\mathscr{Y ~ и ~ г и л ь б е р т о в у ~ п р о с т р а н с т в у ~}$ в случае бесконечномерного $\mathscr{Y}$.

Пусть $y \in \mathscr{Y}$. Положим $g_{s} y=\pi g_{s} z$ для произвольного $z \in \pi^{-1} y$. Рассмотрим кривую $y(s)=g_{s} y$ на $\mathscr{Y}$. 
ЛЕмма 10. Касательный к кривой $y(s)$ вектор отличен от 0.

ДокАЗАТЕЛЬСтво. Предположим противное. Тогда в силу аксиомы $4 \| K^{\varepsilon} g_{\varepsilon s} z-$ $K^{\varepsilon} z \| \rightarrow 0$ при $\varepsilon \rightarrow 0$. В силу аксиомы $2\left\|K^{\varepsilon} g_{\varepsilon s} z-e^{i s} K^{\varepsilon} z\right\| \rightarrow 0$ при $\varepsilon \rightarrow 0$. Поэтому $\left\|e^{i s} K^{\varepsilon} z-K^{\varepsilon} z\right\| \rightarrow 0$ при $\varepsilon \rightarrow 0$. Следовательно, $e^{i s}=1$. Лемма 10 доказана.

СлЕДСтвИЕ. На многообразии Я можно ввести локальные координаты ( $y_{1}$, $\left.y_{2}, \ldots\right)$ такие, ито $g_{s}\left(y_{1}, y_{2}, \ldots\right)=\left(y_{1}+s, y_{2}, \ldots\right)$.

ДокАЗАТЕльство. Пусть $\left(Y_{1}, Y_{2}, \ldots\right)$ - локальные координаты на $\mathscr{Y . ~ П р е о б р а з о - ~}$ вание $g_{s}$ переводит точку $Y$ на $\mathscr{Y}$ с этими локальными координатами в точку с координатами $\left(\left(g_{s} Y\right)_{1},\left(g_{s} Y\right)_{2}, \ldots\right)$. В силу леммы 10 для некоторого $j \partial\left(g_{s} Y\right)_{j} / \partial s \neq 0$; не ограничивая обшности, можно считать, что $\partial\left(g_{s} Y\right)_{1} / \partial s \neq 0$. Рассмотрим отображение $\left(y_{1}, y_{2}, \ldots\right) \mapsto\left(Y_{1}, Y_{2}, \ldots\right)$ вида

$$
Y_{i}\left(y_{1}, y_{2}, y_{3}, \ldots\right)=\left(g_{y_{1}}\left(0, y_{2}, y_{3}, \ldots\right)\right)_{i}, \quad i=\overline{1, \infty} .
$$

При $y_{1}=0$ имеем

$$
\frac{\partial Y_{i}}{\partial y_{1}} \neq 0, \quad \frac{\partial Y_{i}}{\partial y_{j}}=\delta_{i j}, \quad i, j \geqslant 2 .
$$

Поэтому в некоторой окрестности начала координат якобиан перехода от $\left(y_{1}, y_{2}, \ldots\right)$ к $\left(Y_{1}, Y_{2}, \ldots\right)$ отличен от 0 и, таким образом, данное преобразование является обратимым. Следствие доказано.

Таким образом, координаты $\left(y_{2}, y_{3}, \ldots\right)$ являются координатами на пространстве орбит $\mathscr{X}$, т.е. $\mathscr{X}$ является гладким многообразием. Рассмотрим отображение гладких многообразий $g: \mathscr{Y} \rightarrow \mathscr{X}$, сопоставляющее элементу $y \in \mathscr{Y}$ его орбиту $[y]$. Обозначим через $g_{*}$ соответствуюшееотображение касательных к многообразиям $\mathscr{Y}$ и $\mathscr{X}$ в точках $y$ и $[y]$ пространств $g_{*}: T \mathscr{Y}_{y} \rightarrow T \mathscr{X}_{[y]}$.

Пусть $\omega_{n}-$ дифференциальная $n$-форма на $\mathscr{Y}$. Будем говорить, что она допускает peдукиию на пространство орбит $\mathscr{X}$, если существует дифференциальная $n$-форма $\omega_{n}^{x}$ на $\mathscr{X}$ такая, что

$$
\omega_{n}^{x}\left(g_{*} \xi_{1}, \ldots, g_{*} \xi_{n}\right)=\omega_{n}\left(\xi_{1}, \ldots, \xi_{n}\right)
$$

для любых $\xi_{1}, \ldots, \xi_{n} \in T \mathscr{Y}_{y}$.

Исследуем структуру 1-форм $\omega$ и $\Omega$ в локальных координатах $\left(y_{1}, y_{2}, \ldots\right)$.

ЛЕмма 11. Дифференциальные 1-форми $\omega$ и $\Omega$ представляются в виде

$$
\omega=-d y_{1}+\sum_{j \geqslant 2} \omega_{j}\left(y_{2}, y_{3}, \ldots\right) d y_{j}, \quad \Omega=\sum_{j \geqslant 2} \Omega_{j} d y_{j} .
$$

ДокАЗАТЕЛЬСтво. Представим формы $\omega$ и $\Omega$ в виде

$$
\omega=\sum_{j \geqslant 1} \omega_{j} d y_{j}, \quad \Omega=\sum_{j \geqslant 1} \Omega_{j} d y_{j} .
$$


Рассмотрим отображение $\Phi: s \mapsto g_{s} z$. Значение форм $\omega$ и $\Omega$ на касательном векторе $\partial(\pi \Phi) / \partial s$ равны $\omega_{1}$ и $\Omega_{1}$ соответственно. Поэтому в силу теоремы 1

$$
\left\|K^{\varepsilon} \Phi(\alpha \sqrt{\varepsilon}+\beta \varepsilon)-\exp \left(-\frac{i}{\sqrt{\varepsilon}} \omega_{1}(\alpha+\beta \sqrt{\varepsilon})\right) \exp \left(-\frac{i}{2} \frac{\partial \omega_{1}}{\partial y_{1}} \alpha^{2}\right) K^{\varepsilon} e^{i \Omega_{1} \alpha} \Phi(0)\right\| \rightarrow 0, \quad \varepsilon \rightarrow 0 .
$$

С другой стороны, из аксиомы 2 вытекает, что

$$
\left\|K^{\varepsilon} \Phi(\alpha \sqrt{\varepsilon}+\beta \varepsilon)-\exp \left(-\frac{i}{\sqrt{\varepsilon}}(\alpha+\beta \sqrt{\varepsilon})\right) K^{\varepsilon} \Phi(0)\right\| \rightarrow 0, \quad \varepsilon \rightarrow 0 .
$$

Поэтому $\omega_{1}=-1, \Omega_{1}=0$. В силу следствия из леммы 7

$$
0=\left[\Omega_{1}, \Omega_{j}\right]=-i \frac{\partial \omega_{j}}{\partial y_{1}}
$$

Следовательно, $\omega_{j}$ зависят только от $y_{2}, y_{3}, \ldots$ Лемма 11 доказана.

Поскольку для дифференциальной формы $d \omega^{x}$ на $\mathscr{X}$ вида

$$
d \omega^{x}=\sum_{k, j \geqslant 2} \frac{\partial \omega_{j}}{\partial y_{k}} d y_{k} \wedge d y_{j}
$$

справедливо свойство (23), из леммы 11 вытекает

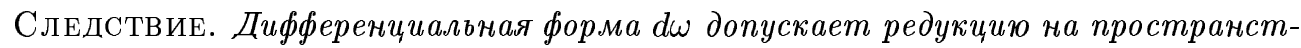
во орбит $\mathscr{X}$.

Таким образом, на фазовом пространстве $\mathscr{X}$ однозначно вводится симплектическая структура $d \omega^{x}$, совпадающая в случае примера 1 с $d P \wedge d Q$. Отметим, что 1-форма действия $\omega$ не допускает редукции на фазовое пространство.

4.2. Примеры нетривиальных фазовых пространств. Рассмотрим примеры абстрактных канонических операторов, которым отвечают топологически нетривиальные фазовые пространства. Эти фазовые пространства возникали в [7].

ПримеР 2. Определим абстрактньй канонический оператор, через который выражаются приближенные решения разностных уравнений. Рассмотрим тривиальное квазиклассическое расслоение $\pi: \mathscr{Z}=\mathscr{Y} \times \mathscr{F} \rightarrow \mathscr{Y}$ со слоем $\mathscr{F}$, являющимся пространством Шварца гладких быстроубывающих функций на прямой, и базой $\mathscr{Y}=\mathbb{R}^{2 n+1}$. Пусть $\varepsilon$ пробегает дискретный набор значений $\{1 / N \mid N \in \mathbb{N}\}$, а $\mathscr{H}^{\varepsilon}$ при $\varepsilon=1 / N$ есть $N$-мерное комплексное пространство. Рассмотрим оператор $K^{\varepsilon}: \mathscr{Y} \times \mathscr{F} \rightarrow \mathscr{H}^{\varepsilon}$ вида

$$
\left(K^{\varepsilon}(S, P, Q ; f)\right)_{k}=\frac{1}{N^{1 / 4}} \sum_{l \in \mathbb{Z}} e^{i N S} \exp \left(i N P\left(\frac{k}{N}-Q-l\right)\right) f\left(\sqrt{N}\left(\frac{k}{N}-Q-l\right)\right)
$$

1-формы $\omega$ и $\Omega$ совпадают с построенными в примере 1. 
ПРИмеР 3. Рассмотренный в примере 2 абстрактный канонический оператор инвариантен относительно дискретной грушшы преобразований базы $\mathscr{Y}:$

$$
P \rightarrow P+2 \pi k, \quad S \rightarrow S+2 \pi Q k+2 \pi n, \quad Q \rightarrow Q+l, \quad k, l, n \in \mathbb{Z} .
$$

Обозначим через $[S, P, Q]$ орбиту точки $(S, P, Q)$, т.е. множество всех точек вида $(25)$, а через $\mathscr{Y}^{\prime}$ соответствующее пространство орбит, также являющееся гладким многообразием. Оператор, сопоставляющий паре $\left(y^{\prime}, f\right), y^{\prime} \in \mathscr{Y}^{\prime}$, где $(S, P, Q)$ - произвольньй представитель класса эквивалентности $y^{\prime}$, удовлетворяет всем аксиомам абстрактного канонического оператора. Отметим, что фазовым пространством в данном случае является тор.

Процедуру построения абстрактного канонического оператора из примера 3 можно обобщить следующим образом. Пусть $K^{\varepsilon}: \mathscr{Z} \rightarrow \mathscr{H}^{\varepsilon}-$ произвольньй абстрактный канонический оператор. Каждому элементу $z \in \mathscr{Z}$ сопоставим класс эквивалентности $[z] \subset \mathscr{Z}$, состоящий из всех $z^{\prime} \in \mathscr{Z}$ таких, что $K^{\varepsilon} z^{\prime}=K^{\varepsilon} z$. Рассмотрим множество всех классов эквивалентности $\mathscr{Z}^{\prime}$ и выберем его в качестве пространства расслоения. База расслоения $\mathscr{Y}^{\prime}$ строится следующим образом. Каждому элементу $y \in \mathscr{Y}$ сопоставим класс эквивалентности $[y]=\bigcup_{z \in \pi^{-1} y} \pi[z]$; $\mathscr{Y}^{\prime}$ является множеством всех таких классов эквивалентности. В качестве отображения $\pi^{\prime}: \mathscr{Z}^{\prime} \rightarrow \mathscr{Y}^{\prime}$ выберем отображение вида $\pi^{\prime}[z]=[\pi z]$, где $z \in[z]$. Положим $K^{\varepsilon \prime}[z]=K^{\varepsilon} z$ для некоторого $z \in[z]$. Данное определение абстрактного канонического оператора $K^{\varepsilon \prime}$ не зависит от выбора представителя класса эквивалентности.

ПРИмеР 4. Параметр $\varepsilon$ может пробегать и непрерьвньй набор значений: можно рассмотреть абстрактньй канонический оператор $K^{\varepsilon}$, переводящий пару $(y, f)$ в вектор (24), отвечающий $N=[1 / \varepsilon]$.

ПРимеР 5. Абстрактньй канонический оператор из примера 4 обобщается на $d$-мерный случай следующим образом. Пусть $\alpha_{1}, \ldots, \alpha_{d} \in \mathbb{R}, \mathscr{F}$ - пространство Шварца гладких быстроубывающих функций на $d$-мерном пространстве, $\pi: \mathscr{Y}_{d} \times \mathscr{F} \rightarrow \mathscr{Y}_{d}-$ тривиальное квазиклассическое расслоение с базой $\mathscr{Y}_{d}=\mathbb{R}^{2 d+1}=\{(S, P, Q) \mid S \in \mathbb{R}$, $\left.P, Q \in \mathbb{R}^{d}\right\}, \mathscr{H}^{\varepsilon}$ - пространство функций целочисленного $d$-мерного вектора $k, i$-я компонента которого пробегает значения от 0 до $N_{i}-1, N_{i}=\left[\alpha_{i} / \varepsilon\right]$. Рассмотрим абстрактный канонический оператор вида

$$
\begin{aligned}
\left(K^{\varepsilon}(S, P, Q ; f)\right)_{k}= & \sum_{l_{1}, \ldots, l_{d} \in \mathbb{Z}} \exp \left(i \sum_{m=1}^{d} N_{m} S+i \sum_{m=1}^{d} N_{m} P_{m}\left(\frac{k_{m}}{N_{m}}-Q_{m}-l_{m}\right)\right) \\
& \times f\left(\sqrt{N_{1}}\left(\frac{k_{1}}{N_{1}}-Q_{1}-l_{1}\right), \ldots, \sqrt{N_{d}}\left(\frac{k_{d}}{N_{d}}-Q_{d}-l_{d}\right)\right) \frac{1}{N^{d / 4}}
\end{aligned}
$$

которому отвечают 1-формы

$$
\omega=\sum_{m=1}^{d} \alpha_{m}\left(P_{m} d Q_{m}-d S\right), \quad \Omega=\sum_{m=1}^{d} \alpha_{m}\left(d P_{m} \xi-d Q_{m} \frac{1}{i} \frac{\partial}{\partial \xi}\right)
$$


Аналогично примеру 3 можно перейти от базы $\mathscr{Y}_{d}$ к базе $\mathscr{Y}_{d}^{\prime}$, состоящей из классов эквивалентности $\left\{\left(S+2 \pi \sum_{m} Q_{m} k_{m}+2 \pi n, P+2 \pi k, Q+l\right)\right\}$, где $k, l$ - целочисленные векторы, $n$ - целое число. Фазовым пространством в данном случае является $d$-мерный Top.

Отметим, что абстрактньй канонический оператор из примера 5 позволяет рассматривать фазовые пространства, не накладывая никаких условий квантования, в отличие от теории асимптотического квантования [7]. Квантованность фазового пространства проявляется здесь в том, что операторы $K^{\varepsilon}$ могут пробегать лиш дискретный набор значений, характеризуемых числами $\left(N_{1}, \ldots, N_{d}\right)$, хотя параметр $\varepsilon$ пробегает непрерьвньй набор значений. Данное замечание аналогично замечанию [7, с. 176], согласно которому любое лагранжево многообразие можно сделать квантованным.

\section{3. Абстрактные канонические операторы, связанные с групшами Ли.}

ПримеР 6. Рассмотрим систему с (целым или полуцелым) угловым моментом $l$, играющим роль "большого" параметра. Пространство $\mathscr{H}^{1 / l}$ выберем в виде комплексного $(2 l+1)$-мерного пространства, т.е. в виде пространства последовательностей $\left(\psi_{-l}\right.$, $\left.\psi_{-l+1}, \ldots, \psi_{l-1}, \psi_{l}\right)$. Абстрактный канонический оператор выражается через оператор $W_{l}$, действующий из комплексного евклидова пространства финитных последовательностей из $\ell^{2}$ в пространство $\mathscr{H}^{1 / l}$, переводящий бесконечную последовательность $\left(f_{0}, f_{1}, \ldots\right)$ в конечную последовательность $\left(\left(W_{l} f\right)_{-l},\left(W_{l} f\right)_{-l+1}, \ldots,\left(W_{l} f\right)_{l}\right)$ вида $\left(W_{l} f\right)_{m}=f_{l-m}$. Расширенное фазовое пространство Я является прямым произведением $\mathbb{R} \times \mathrm{SU}(2)$, т.е. множеством всех упорядоченных пар вида $(S, g)$, где $S \in \mathbb{R}$, $g=\left(\begin{array}{cc}\alpha & \beta \\ -\beta^{*} & \alpha^{*}\end{array}\right) \in \mathrm{SU}(2),|\alpha|^{2}+|\beta|^{2}=1$. Рассмотрим представление $T_{g}^{l}$ групшы $\mathrm{SU}(2)$ в пространстве $\mathscr{H}^{1 / l}$. Известно (см., например, [8]), что операторы $T_{g}^{l}: \mathscr{H}^{1 / l} \rightarrow \mathscr{H}^{1 / l}$ однозначно определяются из соотношений на однородные многочлены степени $2 l$, зависящие от аргументов $x$ и $y$ :

$$
\begin{aligned}
& \sum_{m=-l}^{l}\left(T_{g}^{l} \varphi\right)_{m} \sqrt{\frac{(2 l) !}{(l-m) !(l+m) !}} x^{l+m} y^{l-m} \\
& \quad=\sum_{m=-l}^{l} \varphi_{m} \sqrt{\frac{(2 l) !}{(l-m) !(l+m) !}}\left(\alpha x-\beta^{*} y\right)^{l+m}\left(\beta x+\alpha^{*} y\right)^{l-m} .
\end{aligned}
$$

Отображение $K^{\varepsilon}: \mathbb{R} \times \mathrm{SU}(2) \times \ell^{2} \rightarrow \mathscr{H}^{1 / l}$, удовлетворяющее аксиомам $1-6$, определим следующим образом:

$$
K^{\varepsilon}(S, g, f)=\exp \left(\frac{i}{\varepsilon} S\right) T_{g}^{l} W_{l} f, \quad \varepsilon=\frac{1}{l} .
$$

Для исследования преобразования абстрактного канонического оператора (26) при малом сдвиге $S$ и $g$ рассмотрим вектор $T_{g(\tau)}^{l} W_{l} f$, отвечающий кривой $g(\tau)$ на группе $\mathrm{SU}(2)$, выходящей из точки $g(0)=1$. Представим кривую $g(\tau)$ в виде $g(\tau)=\exp A(\tau)$, где $A(\tau)=\left(\begin{array}{cc}i \xi & \eta \\ -\eta^{*} & -i \xi\end{array}\right)-$ антиэрмитова бесследовая матрица, имеющая порядок $O(\tau)$ при 
$\tau \rightarrow 0$. Оператор $T_{g(\tau)}^{l}$ выражается через представление $T_{A}^{l}(\tau)$ соответствующей алгебры Ли:

$$
\begin{gathered}
T_{g(\tau)}^{l}=\exp \left(T_{A}^{l}(\tau)\right) \\
\left(T_{A}^{l}(\tau) \varphi\right)_{m}=2 i \xi m \varphi_{m}+\eta \sqrt{(l+m)(l-m+1)} \varphi_{m-1}-\eta^{*} \sqrt{(l-m)(l+m+1)} \varphi_{m+1}
\end{gathered}
$$

Используя определение оператора $W_{l}$, получаем $T_{g(\tau)}^{l} W_{l} f=W_{l} \exp \left(\widetilde{T}_{A}^{l}(\tau)\right) f$, где

$$
\left(\widetilde{T}_{A}^{l}(\tau) f\right)_{n}=2 i \xi(l-n) f_{n}+\eta \sqrt{(n+1)(2 l-n)} f_{n+1}-\eta^{*} \sqrt{n(2 l-n+1)} f_{n-1}
$$

Учитьвая, что $l=1 / \varepsilon$, получаем

$$
T_{1+\varepsilon \delta g+O\left(\varepsilon^{2}\right)}^{l} W_{l} f=e^{2 \varepsilon l \delta \alpha} W_{l} f+O(\sqrt{\varepsilon}), \quad T_{1+\sqrt{\varepsilon} \delta g+O(\varepsilon)}^{l} W_{l} f=\text { const } W_{l} e^{B} f
$$

где через $B$ обозначен оператор $B: \ell^{2} \rightarrow \ell^{2}$ вида

$$
(B \varphi)_{n}=\sqrt{2 l \varepsilon} \delta \beta \sqrt{n+1} \varphi_{n+1}-\sqrt{2 l \varepsilon} \delta \beta^{*} \sqrt{n} \varphi_{n-1}
$$

Таким образом, в точке $g=1$-форомы $\omega$ и $\Omega$ имеют вид

$$
\omega=2 i d \alpha-d s, \quad \Omega=-\sqrt{2} i\left(d \beta a^{+}-d \beta^{*} a^{-}\right),
$$

где через $a^{ \pm}$обозначены операторы рождения-уничтожения в пространстве $\ell^{2}[9]$ :

$$
\left(a^{+} f\right)_{n}=\sqrt{n} f_{n-1}, \quad\left(a^{-} f\right)_{n-1}=\sqrt{n} f_{n}
$$

Для нахождения форм $\omega$ и $\Omega$ при произвольном $g$ воспользуемся соотношением

$$
K^{\varepsilon}(S+\delta S, g+\delta g, f)=\exp \left(\frac{i}{\varepsilon}(S+\delta S)\right) T_{g}^{l} K^{\varepsilon}\left(0,1+g^{-1} \delta g, f\right)
$$

Из него вытекает, что значения форм $\omega$ и $\Omega$ в точке $g$ на касательном векторе $\delta g$ равны значениям этих форм в точке $g=1$ на касательном векторе $g^{-1} \delta g$ (иными словами, эти формы левоинвариантны). Отсюда при произвольном $g$ получаем

$$
\omega=2 i\left(\alpha^{*} d \alpha-\beta d \beta^{*}\right)-d S, \quad \Omega=-\sqrt{2} i\left(\left(\alpha^{*} d \beta-\beta d \alpha^{*}\right) a^{+}-\left(\alpha d \beta^{*}-\beta^{*} d \alpha\right) a^{-}\right)
$$

ЗАмЕчАниЕ. Утверждение о левоинвариантности 1-форм $\omega$ и $\Omega$ справедливо и в общем случае, когда абстрактный канонический оператор представляется в виде (26) для произвольной групшы Ли. 
ПРИмеР 7. Рассмотренный в примере 6 абстрактный канонический оператор инвариантен относительно преобразований:

$$
S \rightarrow S+\tau, \quad \alpha \rightarrow \alpha e^{-i \tau / 2}, \quad \beta \rightarrow \beta e^{i \tau / 2}, \quad f \rightarrow e^{-i a^{+} a^{-} \tau} f,
$$

где $\tau$ - вешественньй параметр. Применяя описанную в предыдушем примере процедуру редукции абстрактного канонического оператора, получаем отображение $K^{\varepsilon}$ следующего вида. В качестве пространства $\mathscr{Z}^{\prime}$ рассмотрим факторпространство пространства $\mathscr{Z}$ по отношению эквивалентности (28). Базой $\mathscr{Y}^{\prime}$ является множество всех классов эквивалентности $[S, \alpha, \beta]$. На $\mathscr{Y}^{\prime}$ однозначно определены 1 -формы $\omega$ и $\Omega(27)$. Фазовым пространством является фактормногообразиегруппы $\mathrm{SU}(2)$ по отношению эквивалентности $\alpha \rightarrow \alpha e^{-i \tau / 2}, \beta \rightarrow \beta e^{i \tau / 2}$, т.е. двумерная сфера с симплектической 2-формой, задающей площадь сферы.

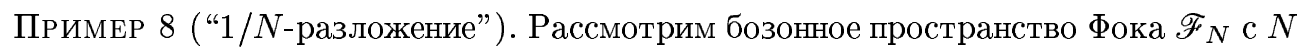
степенями свободы ( $N$ - большой параметр). Напомним [9], что элементами этого пространства являются наборы

$$
\left(f^{(0)}, f_{i_{1}}^{(1)}, f_{i_{1} i_{2}}^{(2)}, \ldots, f_{i_{1} \ldots i_{n}}^{(n)}, \ldots\right)
$$

симметричных по $i_{1}, \ldots, i_{n} \in\{1,2, \ldots, N\}$ функщий $f_{i_{1} \ldots i_{n}}^{(n)}$, удовлетворяющие свойству

$$
\sum_{n=0}^{\infty} \sum_{i_{1}, \ldots, i_{n}=1}^{N}\left|f_{i_{1} \ldots i_{n}}^{(n)}\right|^{2}<\infty
$$

Скалярное произведение в $\mathscr{F}_{N}$ задается следующим образом:

$$
(f, g)=\sum_{n=0}^{\infty} \sum_{i_{1}, \ldots, i_{n}=1}^{N} f_{i_{1} \ldots i_{n}}^{(n) *} g_{i_{1} \ldots i_{n}}^{(n)}
$$

а операторы рождения-уничтожения $a_{1}^{+}, \ldots, a_{N}^{+}, a_{1}^{-}, \ldots, a_{N}^{-}$имеют вид

$$
\left(a_{i}^{+} f\right)_{i_{1} \ldots i_{n}}^{(n)}=\frac{1}{\sqrt{n}} \sum_{k=1}^{n} \delta_{i i_{k}} f_{i_{1} \ldots i_{k-1} i_{k+1} \ldots i_{n}}^{(n-1)}, \quad\left(a_{i}^{-} f\right)_{i_{1} \ldots i_{n-1}}^{(n-1)}=\sqrt{n} f_{i i_{1} \ldots i_{n-1}}^{(n)} .
$$

Вакуумным вектором $\Phi_{N}^{(0)} \in \mathscr{F}_{N}$ называется вектор, нулевая компонента которого равна 1 , а остальные компоненты равны 0. Рассмотрим группу Ли, состоящую из матриц $\left(\begin{array}{cc}u & v \\ v^{*} & u^{*}\end{array}\right),|u|^{2}-|v|^{2}=1$, и ее универсальную накрывающую, которую обозначим

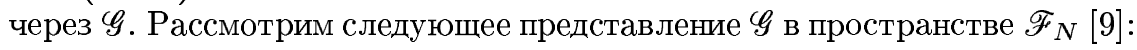

$$
T_{\exp \left(i t\left(\begin{array}{cc}
-c & b \\
b^{*} & c
\end{array}\right)\right)}^{N}=\exp \left(i t\left(\frac{b}{2} \sum_{k=1}^{N}\left(a_{k}^{+}\right)^{2}+\frac{b^{*}}{2} \sum_{k=1}^{N}\left(a_{k}^{-}\right)^{2}+c \sum_{k=1}^{N} a_{k}^{+} a_{k}^{-}+\frac{N c}{2}\right)\right)
$$

и оператор $W_{N}: \mathscr{F}_{1} \rightarrow \mathscr{F}_{N}$ вида

$$
W_{N} f=\sum_{n=0}^{\infty} \frac{1}{\sqrt{n !}} f^{(n)}\left(\frac{1}{\sqrt{2 N}} \sum_{k=1}^{N}\left(a_{k}^{+}\right)^{2}\right)^{n} \Phi_{N}^{(0)}
$$


Рассмотрим расширенное фазовое пространство $\mathscr{Y}=\mathbb{R} \times \mathscr{G}$ и тривиальное расслоение $\pi: \mathscr{Y} \times \mathscr{F} \rightarrow \mathscr{Y}$, где $\mathscr{F}$ - множество всех финитных векторов из $\mathscr{F}_{1}$. Абстрактньй канонический оператор $K^{\varepsilon}: \mathscr{Y} \times \mathscr{F} \rightarrow \mathscr{F}_{N}(\varepsilon=1 / N)$ вида

$$
K^{\varepsilon}(S, g, f)=\exp \left(\frac{i}{\varepsilon} S\right) T_{g}^{1 / \varepsilon} W_{N} f
$$

используется при построении асимптотик в теории большого числа полей с помощью метода $1 / N$-разложения [10], [11]. Отображению (29) отвечают инвариантные 1-формы $\omega$ и $\Omega$, имеющие при $g=1$ вид

$$
\omega=\frac{i}{2 i} d u-d S, \quad \Omega=-\frac{i}{\sqrt{2}}\left(a^{+} d v-a^{-} d v^{*}\right),
$$

а при произвольном $u, v$ вид

$$
\omega=-\frac{i}{2}\left(u^{*} d u-v d v^{*}\right)-d S, \quad \Omega=-\frac{i}{\sqrt{2}}\left(a^{+}\left(u^{*} d v-v d u^{*}\right)-a^{-}\left(u d v^{*}-v^{*} d u\right)\right) .
$$

ПримеР 9. Рассмотренный в примере 8 абстрактный канонический оператор инвариантен относительно преобразования

$$
u \rightarrow u e^{i \tau / 2}, \quad v \rightarrow v e^{-i \tau / 2}, \quad S \rightarrow S+\frac{\tau}{4}, \quad f \rightarrow e^{i \tau a^{+} a^{-}} f .
$$

Применяя процедуру редукции, описанную в подпункте 4.2 , получаем абстрактньй канонический оператор, которому отвечает фазовое пространство, элементами которого являются классы эквивалентности $[(u, v)]=\left\{\left(u e^{i \tau / 2}, v e^{-i \tau / 2}\right)\right\}$. Каждому классу эквивалентности взаимно однозначно сопоставляется число $z=v / u^{*}$, которое по модулю меньше 1 ; числу $z$ сопоставляется класс эквивалентности

$$
\left\{\left(\frac{1}{1-|z|^{2}} e^{i \tau / 2}, \frac{z}{1-|z|^{2}} e^{-i \tau / 2}\right)\right\}
$$

Поскольку симплектическая структура на фазовом пространстве имеет вид

$$
-\frac{i}{2\left(1-|z|^{2}\right)^{2}} d z^{*} \wedge d z
$$

фазовое пространство в данном примере совпадает с плоскостью Лобачевского. Отметим, что фазовые пространства в виде сферы и плоскости Лобачевского рассматривались в [12].

\section{4. Применения к системам многих частищ.}

ПримеР 10. Пусть $\mathscr{F}$ - бозонноепространство Фока с бесконечным числом степеней свободы. Положим $\mathscr{H}^{\varepsilon}=\mathscr{F}, \pi: \mathbb{R} \times \ell^{2} \times \mathscr{F} \rightarrow \mathbb{R} \times \ell^{2}$ - тривиальное расслоение,

$$
K^{\varepsilon}(S, \varphi, f)=\exp \left(\frac{i}{\varepsilon} S\right) \exp \left(\frac{1}{\sqrt{\varepsilon}} \sum_{k=1}^{\infty}\left(\varphi_{k} a_{k}^{+}-\varphi_{k}^{*} a_{k}^{-}\right)\right) f .
$$

Данному абстрактному каноническому оператору отвечают 1-формы

$$
\omega=\frac{i}{2} \sum_{k=1}^{\infty}\left(\varphi_{k}^{*} d \varphi_{k}-\varphi_{k} d \varphi_{k}^{*}\right)-d S, \quad \Omega=\sum_{k=1}^{\infty}\left(d \varphi_{k} a_{k}^{+}-d \varphi_{k}^{*} a_{k}^{-}\right) .
$$


ПРИмеР 11 ("многочастичный канонический оператор"). Пусть $\mathscr{X}$-множество всех векторов (комплексного) пространства $\ell^{2}$ с единичной нормой, $\mathscr{H}^{\varepsilon}, \varepsilon=1 / N,-$ пространство симметричньх функций $f_{i_{1} \ldots i_{N}}\left(i_{1}, \ldots, i_{N}=\overline{1, \infty}\right)$ с суммируемым квадратом: $\sum_{i_{1} \ldots i_{N}}\left|f_{i_{1} \ldots i_{N}}^{(N)}\right|^{2}<\infty ; \pi: \mathbb{R} \times \mathscr{X} \times \mathscr{F} \rightarrow \mathbb{R} \times \mathscr{X}$ - тривиальное расслоение. Рассмотренному в [2] абстрактному каноническому оператору

$$
\left(K^{\varepsilon}(S, \varphi, f)_{i_{1} \ldots i_{N}}=\sum_{p=0}^{N} \frac{1}{\sqrt{N^{p} p !}} \sum_{1 \leqslant k_{1} \neq \cdots \neq k_{p} \leqslant N} f_{k_{1} \ldots k_{p}}^{p} \prod_{k \neq k_{1}, \ldots, k_{p}} \varphi_{k}\right.
$$

также отвечают формы (30).

Отметим, что многочастичный канонический оператор (31) обладает свойством инвариантности

$$
\varphi \rightarrow \varphi e^{i \alpha}, \quad S \rightarrow S-\alpha, f \rightarrow e^{i \alpha a^{+} a^{-}} f,
$$

что позволяет построить редуцированньй канонический оператор, которому отвечает фазовое пространство, являющееся проективным пространством, состоящим из одномерных подпространств пространства $\ell^{2}$.

Автор благодарит академика РАН В.П. Маслова за полезное обсуждение ряда вопросов и ценные замечания.

\section{СПИСОК ЦИТИРОВАННОЙ ЛИТЕРАТУРЫ}

[1] Маслов В. П. Комплексный метод ВКБ в нелинейных уравнениях. М.: Наука, 1977.

[2] Maslov V.P., Shvedov O. Yu. The chaos conservation problem in quantum physics // Russian J. Math. Phys. 1996. V. 4. № 2. P. 173-216.

[3] Маслов В.П., Шведов О. Ю. Асимптотика решения уравнения Шрёдингера для спиновых систем // Докл. РАН. 1998. Т. 361. № 4. С. 453-457.

[4] Маслов В. П., Шведов О. Ю. Комплексный метод ВКБ в пространстве Фока // Докл. PAH. 1995. T. 340. № 1 . C. 42-47.

[5] Маслов В.П., Шведов О. Ю. О проблеме расходимости в квантовой теории поля // Докл. РАН. 1997. Т. 357. № 5. С. 604-608.

[6] Маслов В.П., Шведов О.Ю. О некоторых тождествах для асимптотик решений абстрактных уравнений // Докл. РАН. 1997. Т. 352. № 2. С. 167-171.

[7] Карасёв М. В., Маслов В. П. Нелинейная скобка Пуассона. Геометрия и квантование. М.: Наука, 1991.

[8] ЖКелобенко Д. П., Штерн А. И. Представления групп Ли. М.: Наука, 1983.

[9] Березин Ф. А. Метод вторичного квантования. М.: Наука, 1986.

[10] Berezin F. A. Models of Gross-Neveu type are quantization of a classical mechanics with nonlinear phase space // Comm. Math. Phys. 1978. V. 63. P. 131-153.

[11] Yaffe L. G. Large $N$ limits as classical mechanics // Rev. Modern Phys. 1982. V. 54. № 2. P. 407-436.

[12] Berezin F. A. General concept of quantization // Comm. Math. Phys. 1975. V. 40. № 2. P. 153-174.

Московский государственный университет им. М.В. Ломоносова

Поступило

E-mail: shvedov@qs.phys.msu.su 